\title{
Correction to: Heritabilities and genetic correlations for honey yield, gentleness, calmness and swarming behaviour in Austrian honey bees
}

\author{
Evert W. Brascamp ${ }^{1}$, Alfons Willam ${ }^{2}$, Christian BoIGENZAHN $^{3}$, Piter BiJMA ${ }^{1}$, \\ Roel F. VeERKAMP ${ }^{4}$ \\ ${ }^{1}$ Animal Breeding and Genomics Centre, Wageningen University, Wageningen, The Netherlands \\ ${ }^{2}$ Division of Livestock Sciences, University of Natural Resources and Life Sciences (BOKU), Vienna, Austria \\ ${ }^{3}$ Biene Österreich, Vienna, Austria \\ ${ }^{4}$ Animal Breeding and Genomics Centre, Livestock Research, Wageningen, The Netherlands
}

\section{Correction to: Apidologie, 2016 \\ https://doi.org/10.1007/s13592-016-0427-9}

The original article shows a statistical model that included both the worker effect of colonies and queen effect of queens in the colonies. However, we would like to note that we estimated the phenotypic variances incorrectly. This error became clear after analyzing data with models only including either worker effect of colonies or queen effect of queens. It then appeared that the estimates of the phenotypic variances between the latter two models differed greatly because we incorrectly computed this variance in the model with worker effect of colonies. This correction brings the aforementioned to attention and contains an adjusted Table 1 and explanation of the backgrounds.

In the paper, we computed the phenotypic variances of traits ignoring that the worker effect is in fact the colony mean, which has consequences for the estimates of heritabilities. The erratum involves Table I with estimated

Corresponding author: E. Brascamp,

pim.brascamp@wur.nl

The online version of the original article can be found at (https://doi.org/10.1007/s13592-016-0427-9) genetic parameters. The correction has no consequences for other elements of the paper, like the estimates of the genetic correlations between traits or the estimation of breeding values or their validation.

The table should read as follows.

The phenotypic variance includes the variance for worker effect of colonies, which equals 0.317 Var (worker) as it is the variance of a mean; the figure 0.317 equals the average additive genetic relationship between workers in the base population, and can be computed from Eq. (24a) as referred to in the Appendix of the paper. As an example, for honey yield, the phenotypic variance Var (phenotype) equals $0.317 \times 334.1+173.6-190.3+160.1=$ 249.3. The heritability for worker effect was calculated as the fraction of the phenotypic variance that can be attributed to colonies and equals 0.317 Var (worker)/Var (phenotype).

In this paper, the statistical model included both the worker effect of colonies and queen effect of queens in the colonies. Our error became clear after we analysed data with models only including either worker effect of colonies or queen effect of queens. It appeared that the estimates of the phenotypic variances between the latter two models differed greatly, in retrospect because we incorrectly computed this variance in the model with worker effect of colonies. 
Table I. Estimated genetic parameters. Variances (Var) of worker and queen effect, their covariance and residual and phenotypic variances. Derived from these are variance for selection criterion $\left(\sigma_{S C}^{2}\right)$, estimates of heritabilities for worker effect $\left(h_{W}^{2}\right)$, queen effect $\left(h_{Q}^{2}\right)$, genetic correlation between worker and queen effect $\left(r_{g}\right)$ and heritabilities for selection criterion $\left(h_{S C}^{2}\right)$. Approximate standard errors are given in brackets.

\begin{tabular}{lcccr}
\hline & Honey yield (kg) & $\begin{array}{l}\text { Gentleness } \\
(1-4)\end{array}$ & $\begin{array}{l}\text { Calmness } \\
(1-4)\end{array}$ & $\begin{array}{l}\text { Swarming behaviour } \\
(1-4)\end{array}$ \\
\hline Var (worker) & $334.1(62.2)$ & $0.112(0.028)$ & $0.112(0.026)$ & $0.255(0.066)$ \\
Var (queen) & $173.6(35.9)$ & $0.037(0.016)$ & $0.019(0.012)$ & $0.176(0.043)$ \\
Covariance & $-190.3(45.2)$ & $-0.024(0.018)$ & $-0.017(0.014)$ & $-0.195(0.052)$ \\
Var (residual) & $160.1(6.3)$ & $0.148(0.005)$ & $0.146(0.004)$ & $0.467(0.011)$ \\
Var (phenotype) & $249.3(3.9)$ & $0.196(0.009)$ & $0.184(0.003)$ & $0.529(0.043)$ \\
$\sigma_{S C}^{2}$ & $127.1(29.3)$ & $0.10(0.02)$ & $0.10(0.02)$ & $0.04(0.03)$ \\
$h_{W}^{2}$ & $0.42(0.08)$ & $0.18(0.04)$ & $0.19(0.04)$ & $0.15(0.04)$ \\
$h_{Q}^{2}$ & $0.70(0.14)$ & $0.19(0.08)$ & $0.10(0.06)$ & $0.33(0.08)$ \\
$r_{G}$ & $-0.79(0.06)$ & $-0.38(0.19)$ & $-0.36(0.20)$ & $-0.92(0.06)$ \\
$h_{S C}^{2}$ & $0.51(0.12)$ & $0.51(0.10)$ & $0.53(0.10)$ & $0.08(0.05)$ \\
\hline
\end{tabular}

